\title{
Latest measurements in single top quark with the CMS experiment
}

\author{
Fabio lemmi* on behalf of the CMS collaboration \\ Alma Mater Studiorum - Università di Bologna (Unibo) \\ Istituto Nazionale di Fisica Nucleare (INFN) \\ E-mail: fabio.iemmi@cern.ch
}

The latest measurements obtained by the CMS Collaboration in single top quark production are summarized. We present both inclusive and differential cross section measurements in different production modes, which lead to constraints on proton parton distribution functions (PDFs) and measurements of the Cabibbo-Kobayashi-Maskawa (CKM) matrix element $\left|\mathrm{V}_{\mathrm{tb}}\right|$ and top quark spin asymmetry $\mathrm{A}_{\ell}$; we also show searches for rare Standard Model (SM) processes, such as the first observation of $\mathrm{tZq}$ associated production and the first evidence for $\mathrm{t} \gamma \mathrm{q}$ associated production.

7th Annual Conference on Large Hadron Collider Physics - LHCP2019

20-25 May, 2019

Puebla, Mexico

${ }^{*}$ Speaker. 


\section{Introduction}

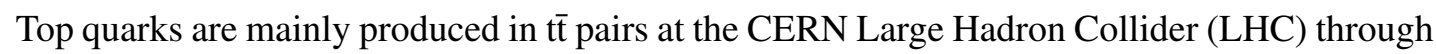
the strong interaction $\left(\sigma_{\mathrm{tt}}^{13} \mathrm{TeV} \approx 830 \mathrm{pb}\right)$. Single top quark production is rarer but better understood from the theoretical point of view, since it's mediated by the weak interaction. Three single top quark production modes are possible at the LHC:

1. $t$-channel: exchange of a space-like $\mathrm{W}$ boson $\left(\sigma_{t \text {-ch }}^{13 \mathrm{TeV}} \approx 220 \mathrm{pb}\right)$;

2. $\mathrm{tW}$ production: associated production with an on-shell $\mathrm{W}$ boson $\left(\sigma_{\mathrm{tW}}^{13 \mathrm{TeV}} \approx 70 \mathrm{pb}\right)$;

3. $s$-channel: exchange of a time-like $\mathrm{W}$ boson $\left(\sigma_{s-\mathrm{ch}}^{13 \mathrm{TeV}} \approx 10 \mathrm{pb}\right)$.

Leading order Feynman diagrams for each of these processes are reported in Figure 1.

Thank to the high integrated luminosity developed by the LHC we are now entering the precision era in $t$-channel measurements, where it is also possible to exploit the distinctive signature of a forward jet coming from the light quark recoiling against the $\mathrm{W}$ boson.

All the presented measurements are performed with data collected by the CMS experiment in 2016, corresponding to an integrated luminosity of $35.9 \mathrm{fb}^{-1}$, unless otherwise stated.
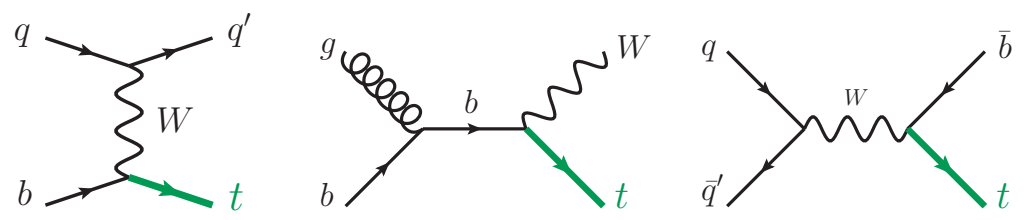

Figure 1: Leading order Feynman diagrams for $t$-channel (left), tW (center) and $s$-channel (right) production modes.

\section{Inclusive and differential measurements}

In this section we present the latest CMS results for inclusive and differential cross section measurements in different production modes.

\subsection{Single $t$ and $\bar{t}$ cross section ratio and $V_{t b}$}

This measurement [1] targets $t$-channel single top quark events. The flavor of the initial light quark determines the charge of the resulting top quark: up quarks in the initial state result in top quarks, while down quarks produce top antiquarks. Since charge asymmetry is present inside protons, the ratio of the cross sections for these two processes, $\mathrm{R}_{t \text {-ch }}=\sigma_{t-\mathrm{ch}, \mathrm{t}} / \sigma_{t-\mathrm{ch}, \mathrm{t}}$, is expected to be different from one. It also provides insight into the inner structure of protons as described by the PDFs. Moreover, a measurement of the total cross section $\sigma_{t-\mathrm{ch}, \mathrm{t}+\overline{\mathrm{t}}}$ allows to perform a direct measurement of the CKM matrix element $\left|f_{\mathrm{LV}} \mathrm{V}_{\mathrm{tb}}\right|$ exploiting the relation $\left|f_{\mathrm{LV}} \mathrm{V}_{t b}\right|=\sqrt{\frac{\sigma_{t-\mathrm{ch}, t+\overline{\mathrm{t}}}}{\sigma_{t-\mathrm{ch}, t+\mathrm{i}}^{\mathrm{th}}}}$, where the anomalous form factor $f_{\mathrm{LV}}$ takes into account the possible presence of an anomalous Wtb coupling and is assumed to be exactly one in the SM framework. 
The analysis selects leptonic top quark decays, namely events containing a single isolated muon or electron in the final state. These leptons originate from the decay of the $\mathrm{W}$ boson coming from the top quark decay, either directly or via the $\mathrm{W} \rightarrow \tau v$ and $\tau \rightarrow \ell v$ decays. Given the $t$-channel topology, the signal signature is one charged, isolated lepton, $\mathrm{p}_{\mathrm{T}}$ imbalance, and two jets, one of which is $\mathrm{b}$ tagged. A second $\mathrm{b}$ jet, arising in the production process via gluon splitting (4 flavor scheme, $4 \mathrm{FS})$, generally has a softer $\mathrm{p}_{\mathrm{T}}$ spectrum and a broader pseudorapidity $(\eta)$ distribution compared to the $\mathrm{b}$ jet coming from the top quark decay, and usually escapes detection. Events containing additional leptons are rejected.

The events used in the analysis are divided into orthogonal categories depending on the number of selected jets and the number of $\mathrm{b}$ tagged jets (njets-mtags regions). Events in the 2 jets-1tag category form the signal region, while events in the 3jets-1tag and 3jets-2tags categories form control regions used to constrain the main background of the analysis, namely t $\bar{t}$ production.

In order to extract the signal, boosted decision trees (BDT) algorithms are employed to combine multiple variables into a single discriminator, enhancing the separation between signal and background processes. Important variables to achieve this task are the light quark $|\eta|$, the reconstructed top quark mass, the invariant mass of the dijet system and the distance in the $\eta-\phi$ plane between the lepton and the $\mathrm{b}$ tagged jet. The signal is extracted by performing a simultaneous, maximum-likelihood (ML) fit to the BDT distributions for each region, lepton flavor and lepton

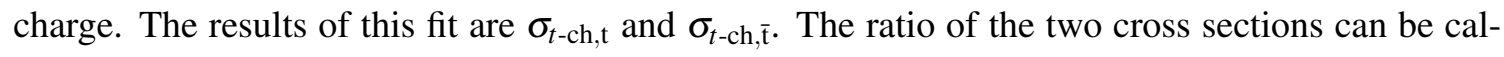
culated from these two results propagating their uncertainties using the covariance matrix coming from the fit. Nonetheless, a more elegant and straightforward way to properly account for the correlations between uncertainties in the cross sections is to repeat the fit with one of the two cross sections replaced by their ratio. This way, potential cancellations of uncertainties are taken into account directly in the fit and do not need to be computed afterwards.

Several sources of systematic uncertainty are considered, either as nuisance parameters in the ML fit (profiled uncertainties) or as nonprofiled uncertainties. The impact of the latter is determined by repeating the analysis using varied templates according to the systematic uncertainties under study instead of the nominal templates. The dominant systematic uncertainty is found to be the one concerning the signal model (PDFs, parton shower scale, etc.).

The resulting value for the ratio $\mathrm{R}_{t-\mathrm{ch}}$ is found to be

$$
\mathrm{R}_{t-\mathrm{ch}}=1.66 \pm 0.02 \text { (stat) } \pm 0.05 \text { (syst) }
$$

in good agreement with most of the predictions, as showed in Figure 2. The measured total cross section translates into

$$
\left|f_{\mathrm{LV}} \mathrm{V}_{\mathrm{tb}}\right|=1.00 \pm 0.08(\exp ) \pm 0.02 \text { (theo) }
$$

where the first uncertainty considers all uncertainties of the measured total cross section, while the second uncertainty is derived from the uncertainty of the theoretical SM prediction. The measured value is in agreement with the assumption of $\left|\mathrm{V}_{\mathrm{tb}}\right|=1$ used to compute the theoretical cross section.

\subsection{ATLAS and CMS combination at $\sqrt{s}=7$ and $8 \mathrm{TeV}$}

Regarding the single top quark cross sections and $\left|f_{\mathrm{LV}} \mathrm{V}_{\mathrm{tb}}\right|$ measurements, a combination of ATLAS and CMS results has been performed [2]. The $t$-channel, tW, and $s$-channel single top 


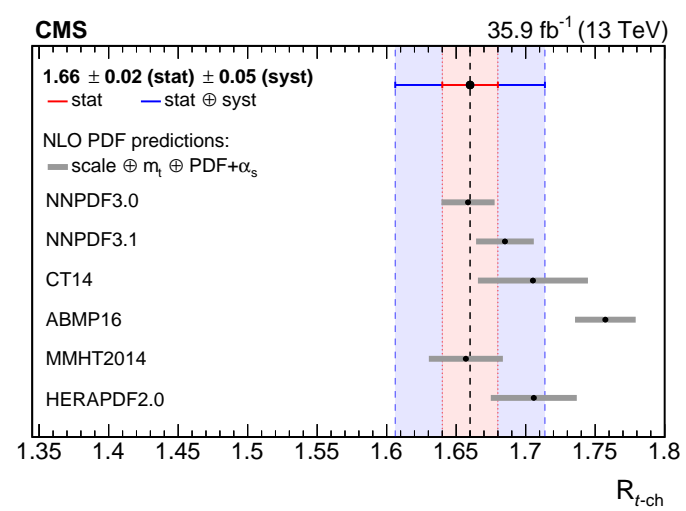

Figure 2: Comparison of the measured $\mathrm{R}_{t \text {-ch }}$ (central, dashed line) with the next-to-leading order (NLO) predictions for different PDFs sets. For the measurement, statistical and systematic uncertainties are indicated individually by the inner and outer error bars.

quark cross section measurements by ATLAS and CMS are combined for each production mode, separately at centre-of-mass energies of 7 and $8 \mathrm{TeV}$, corresponding to integrated luminosities of 1.17 to $5.1 \mathrm{fb}^{-1}$ at $\sqrt{s}=7 \mathrm{TeV}$ and 12.2 to $20.3 \mathrm{fb}^{-1}$ at $\sqrt{s}=8 \mathrm{TeV}$. A combined determination of $\left|f_{\mathrm{LV}} \mathrm{V}_{\mathrm{tb}}\right|$ is also computed.

The combination is performed through iterative methods, making assumptions about correlations between similar sources of uncertainty in different measurements. All the cross section measurements are found to be in good agreement with their corresponding theoretical predictions within uncertainties, as shown in Figure 3. The combined $\left|f_{\mathrm{LV}} \mathrm{V}_{\mathrm{tb}}\right|$ value from the cross section measurements at $\sqrt{s}=7$ and $8 \mathrm{TeV}$, including uncertainties in $\sigma_{\text {theo }}$ for each production mode, is found to be

$$
\left|f_{\mathrm{LV}} \mathrm{V}_{\mathrm{tb}}\right|=1.02 \pm 0.04 \text { (meas) } \pm 0.02 \text { (theo), }
$$

resulting in a relative uncertainty of $3.7 \%$, which improves on the precision of $4.7 \%$ of the most precise individual $\left|f_{\mathrm{LV}} \mathrm{V}_{\mathrm{tb}}\right|$ extraction (ATLAS, $t$-channel analysis at $\sqrt{s}=8 \mathrm{TeV}$ [3]). A summary of the $\left|f_{\mathrm{LV}} \mathrm{V}_{\mathrm{tb}}\right|$ combinations is shown in Figure 3.

\subsection{Differential cross sections and charge ratios, spin asymmetry $\mathbf{A}_{\ell}$}

The CMS collaboration also performed a measurement [4] of differential cross sections for $t$ channel single top quark production in the 4FS (see Figure 4), targeting leptonic top quark decays. In addition, charge ratios, defined as the ratio of the single top quark cross section to the sum of the single top quark and antiquark cross sections, are measured differentially. These quantities are sensitive to proton PDFs and can be used to set constraints on them. Furthermore, differential angular distributions can be used to assess the electroweak coupling structure of the Wtb vertex, where a vector-axial vector (V-A) coupling is predicted in the SM. A powerful observable to investigate the nature of the coupling structure in the $t$-channel is the top quark polarization angle $\cos \theta_{\text {pol. }}^{*}$, defined as

$$
\cos \theta_{\text {pol. }}^{*}=\frac{\vec{p}_{\mathrm{q}^{\prime}}^{\text {(top) }} \cdot \vec{p}_{\ell}^{\text {(top) }}}{\left|\vec{p}_{\mathrm{q}^{\prime}}^{\text {(top) }}\right| \cdot\left|\vec{p}_{\ell}^{\text {(top })}\right|},
$$



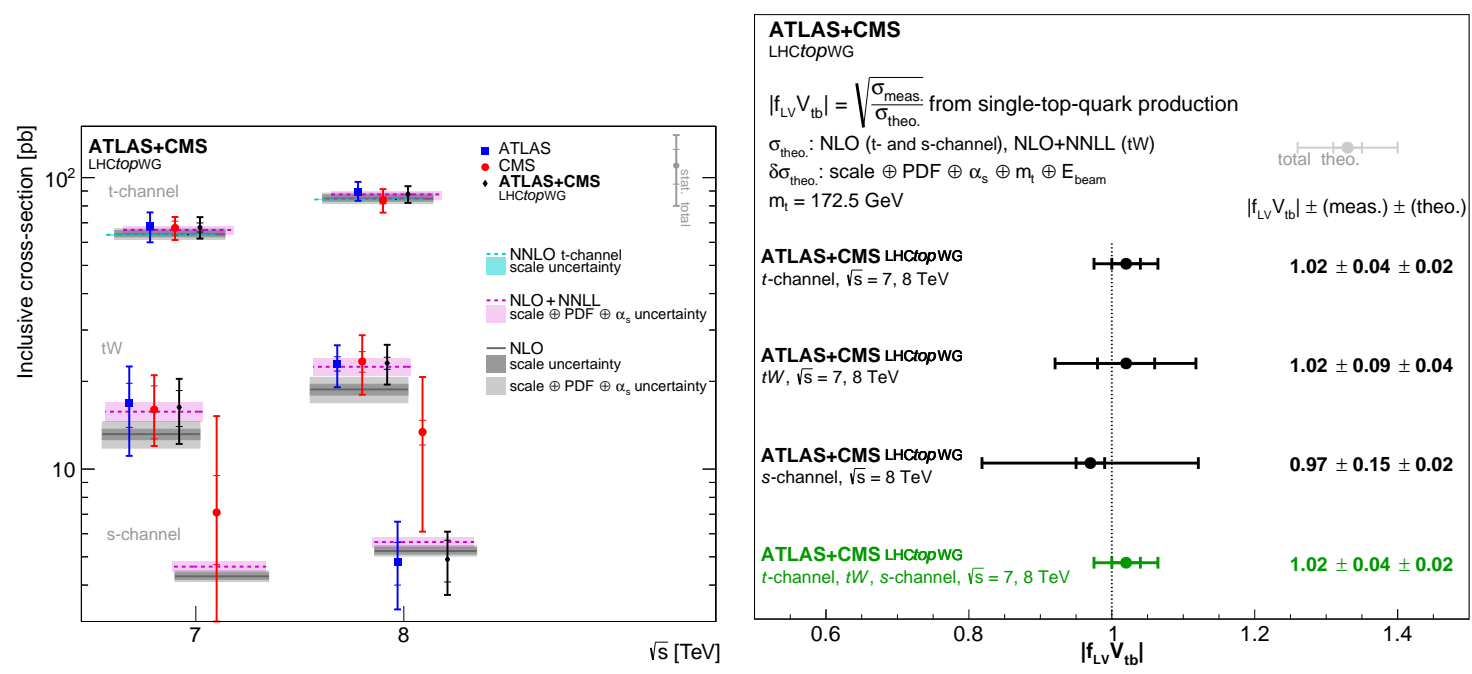

Figure 3: Comparison of the measurements performed by ATLAS and CMS and their combination with the theoretical predictions (left panel); $\left|f_{\mathrm{LV}} \mathrm{V}_{\mathrm{tb}}\right|$ combined measurements along with theoretical and experimental uncertainties (right panel).

where the momenta of the charged lepton, $\ell \in\{\mathrm{e}, \mu\}$, and of the spectator quark, $\mathrm{q}^{\prime}$, are calculated in the top quark rest frame. The normalized differential cross section as a function of $\cos \theta_{\text {pol. }}^{*}$ at parton level is related to the top quark spin asymmetry $\mathrm{A}_{\ell}$ and to the top quark polarization $P$, as

$$
\frac{1}{\sigma} \frac{\mathrm{d} \sigma}{\mathrm{d} \cos \theta_{\mathrm{pol} .}^{*}}=\frac{1}{2}\left(1+2 A_{\ell} \cos \theta_{\mathrm{pol}}^{*}\right), \quad A_{\ell}=\frac{1}{2} P \cdot \alpha_{\ell},
$$

where $\alpha_{\ell}$ is the so-called spin-analyzing power of the charged lepton.

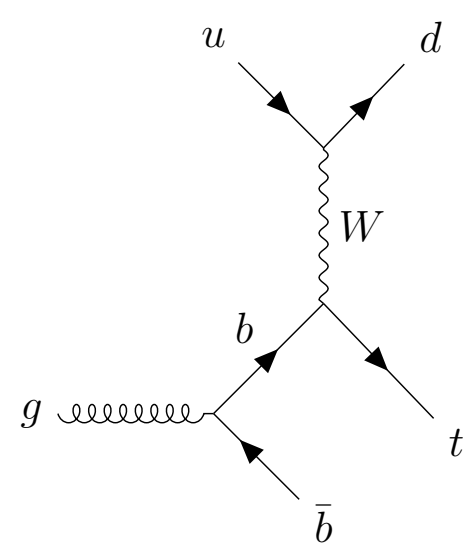

Figure 4: Feynman diagram for single top quark production in the $t$-channel assuming the 4FS.

Given the $t$-channel topology, the signal signature requires one charged, isolated lepton, $\mathrm{p}_{\mathrm{T}}$ imbalance, and two jets, one of which is $\mathrm{b}$ tagged. Events containing additional leptons are rejected. To classify signal and control regions, different orthogonal event categories are defined, denoted $n$ jets-mtags, based on the number of jets and $b$ tagged jets. The total number of jets $n$ can range from 2 to 3 , while the number of $\mathrm{b}$ tagged jets can range from 0 to 2 . The 2 jets- 1 tag category forms the signal region, while the remaining regions are used to assess the background modeling.

The number of $t$-channel single top quark events in data is determined from a ML fit to the $\mathrm{W}$ boson transverse mass distribution $\mathrm{m}_{\mathrm{T}}(\mathrm{W})$ and two $\mathrm{BDT}$ discriminants distributions. The first BDT, labelled $\mathrm{BDT}_{t \text {-ch }}$, is trained to separate $t$-channel events from background events, namely $t \bar{t}, \mathrm{~W}+\mathrm{jets}$ and multijet events. Since the $\mathrm{BDT}_{t-\mathrm{ch}}$ discriminant shapes for the $\mathrm{t} \overline{\mathrm{t}}$ and $\mathrm{W}+\mathrm{jets}$ are found to be similar, to obtain sensitivity in the fit to both backgrounds individually, a second $\mathrm{BDT}$, labelled $\mathrm{BDT}_{\mathrm{tt} / \mathrm{W}}$, is trained to classify events for these two processes only. The ML fit is performed using the following distributions, which are also reported in Figure 5: 
- $\mathrm{BDT}_{t \text {-ch }}$ in the 2jets-1tag region for events with $\mathrm{m}_{\mathrm{T}}(\mathrm{W})>50 \mathrm{GeV}$ and $\mathrm{BDT}_{t \text {-ch }}>0$, a region of the phase space which is enriched in signal events;

- $\mathrm{BDT}_{\mathrm{tt} / \mathrm{W}}$ in the 2jets-1tag region for events with $\mathrm{m}_{\mathrm{T}}(\mathrm{W})>50 \mathrm{GeV}$ and $\mathrm{BDT}_{t-\mathrm{ch}}<0$, a region of the phase space enriched in $\mathrm{t} \overline{\mathrm{t}}$ and $\mathrm{W}+$ jets events but depleted of signal and multijet events;

- $\mathrm{m}_{\mathrm{T}}(\mathrm{W})$ in the 2jets-1 tag region for events with $\mathrm{m}_{\mathrm{T}}(\mathrm{W})<50 \mathrm{GeV}$, a region of the phase space which is particularly sensitive to the amount of multijet events;

- $\mathrm{m}_{\mathrm{T}}(\mathrm{W})$ in the 3 jets-2tags region, a region of the phase space which provides additional sensitivity to the main $t \bar{t}$ background.
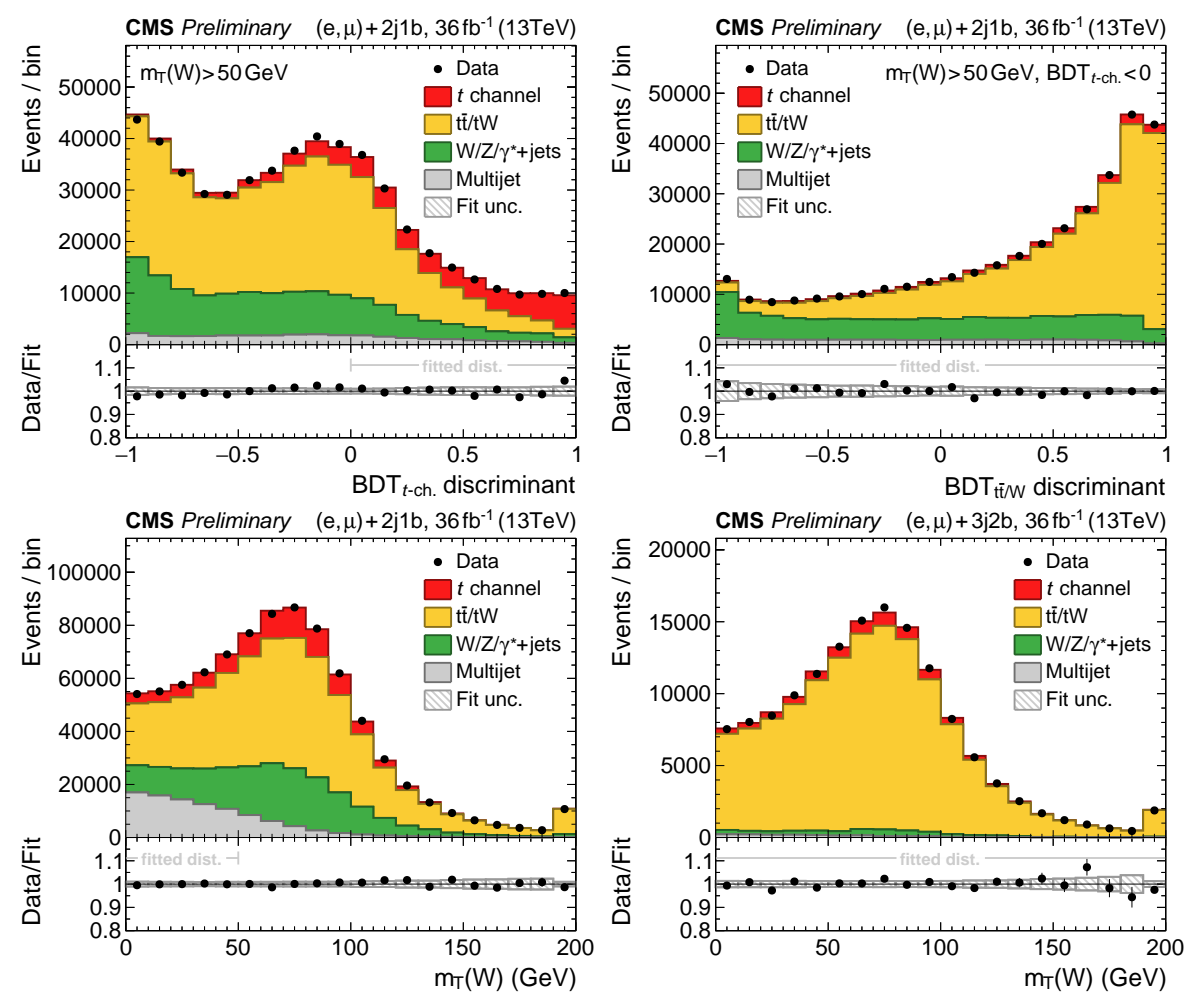

Figure 5: Distributions used in the ML fit to extract the signal yields. $\mathrm{BDT}_{t \text {-ch }}$ distribution in the 2jets1 tag region (upper left panel); $\mathrm{BDT}_{\mathrm{t}} / \mathrm{W}$ distribution in the 2jets-1tag region (upper right panel); $\mathrm{m}_{\mathrm{T}}(\mathrm{W}$ ) distribution in the 2jets-1tag region (lower left panel); $\mathrm{m}_{\mathrm{T}}(\mathrm{W})$ distribution in the 3jets-2tags region (lower right panel).

In order to compute differential cross sections, individual fits are performed for each observable that is later used in the unfolding. In each fit, the signal and background yields can vary independently across the unfolding intervals. The likelihood to be maximized in such fits can be expressed as a product of Poisson-like terms:

$$
\ln L=\sum_{k}^{\text {region interval bins }} \sum_{j} \sum_{i}\left(d_{k j i} \cdot \ln p_{k j i}-p_{k j i}\right),
$$


where $d$ denotes the number of observed events in data and $p$ the estimated prediction. Summation over $k$ denotes the 16 regions (4 distributions $\times$ lepton flavor $\times$ lepton charge), $j$ denotes the interval in the unfolding observable and $i$ denotes the distribution bin. The obtained yields are used to perform unfolding at parton and particle level using the TUNFOLD algorithm [5].

Several systematic uncertainties are taken into account, the uncertainty on the top quark mass being the dominant one.

As a result, the differential charge ratios are found to be in agreement with the theoretical predictions within uncertainties. Also, the 4FS prediction for the differential cross sections is found to be better in agreement with the predictions with respect to the five flavor scheme (5FS, where the $\mathrm{b}$ quark is expected to come from the proton), especially for the top quark and $\mathrm{W}$ boson transverse momenta distributions. Finally, performing a $\chi^{2}$-based fit to the normalized, differential cross section as a function of the top quark polarization angle, the top quark spin asymmetry $\mathrm{A}_{\ell}$ is extracted, which is found to be

$$
\mathrm{A}_{\ell}=0.439 \pm 0.032(\text { stat }+\exp ) \pm 0.053(\text { syst })
$$

which is in good agreement with the theoretical expectations. These results are summarized in Figure 6.
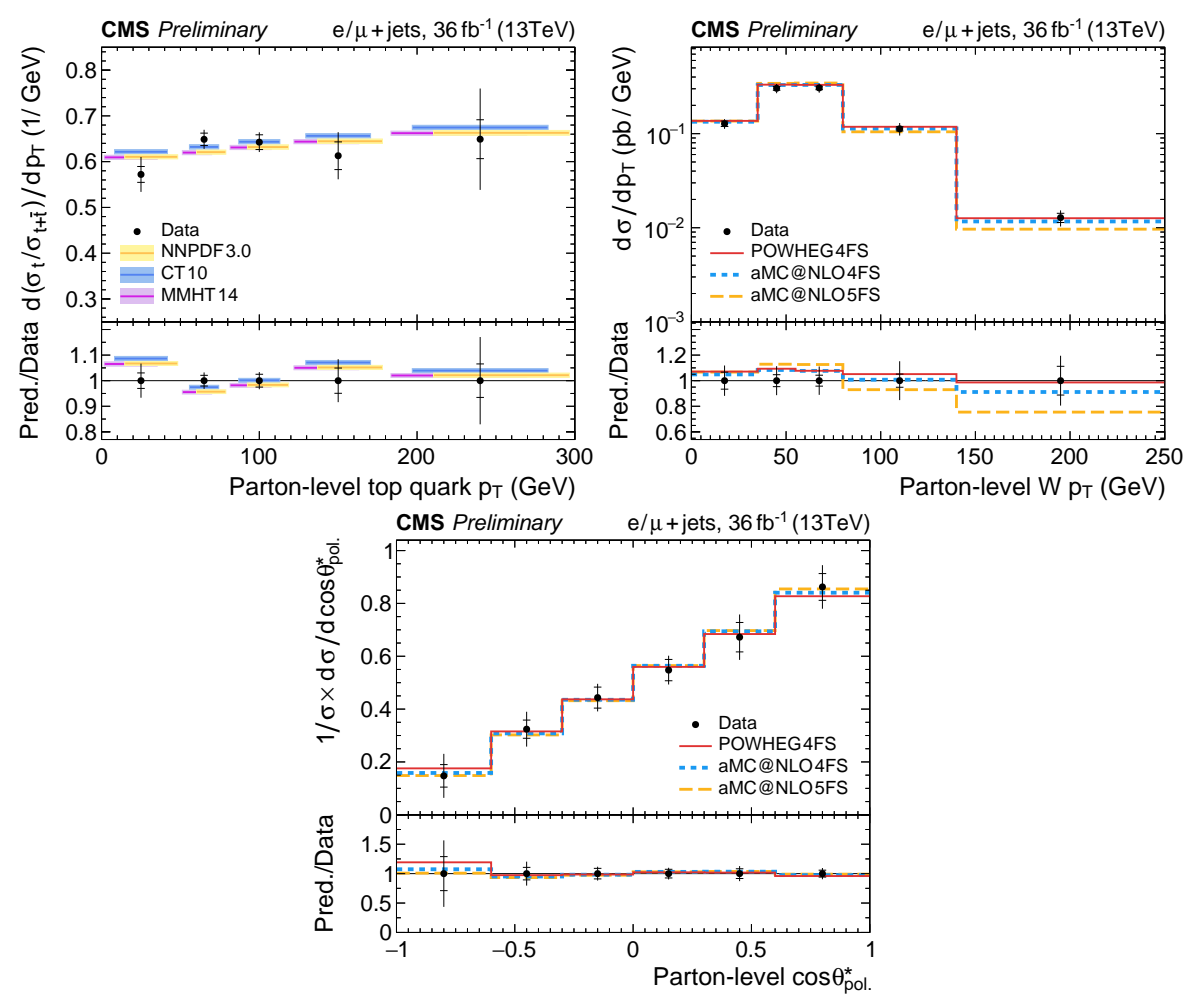

Figure 6: Differential charge ratio as a function of the parton-level top quark $\mathrm{p}_{\mathrm{T}}$ (upper left panel); differential cross section as a function of the parton-level $\mathrm{W}$ boson $\mathrm{p}_{\mathrm{T}}$ (upper right panel); normalized, differential cross section as a function of the parton-level top quark polarization angle used to extract $\mathrm{A}_{\ell}$ (lower panel). 


\section{4 tW production cross section}

The CMS Collaboration also produced the fist measurement of the tW production cross section at $13 \mathrm{TeV}$ [6]. The analysis is performed using the $\mathrm{e}^{ \pm} \mu^{\mp}$ dilepton channel, in which both $\mathrm{W}$ bosons, either produced in association with the top quark or from the decay of the top quark, decay leptonically. Based on the tW topology (see Figure 7), this leads to a final state composed of two, oppositely charged leptons, $\mathrm{p}_{\mathrm{T}}$ imbalance and a $\mathrm{b}$ tagged jet.

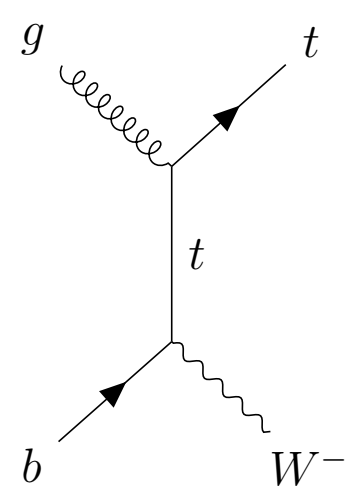

Figure 7: One possible Feynman diagram for the tW associated production.

Events are classified into three orthogonal categories: a signalenriched region with 1jet-1tag, and two background-dominated regions with two jets: a 2jets-1tag region and a 2jets-2tags region. A multivariate method is used to discriminate the tW signal from the main background of the measurement, namely $t \bar{t}$. Several observables are combined into a single discriminator using a BDT technique. Two BDTs are trained, the first one, $\mathrm{BDT}_{1 \mathrm{j}-1 \mathrm{~b}}$ in the $1 \mathrm{jet}-1 \mathrm{tag}$ region and the second one, $\mathrm{BDT}_{2 \mathrm{j}-1 \mathrm{~b}}$, in the $2 \mathrm{jets}-1$ tag region. The signal is extracted by performing a ML fit to the BDT distributions in the corresponding regions, and to the subleading jet $\mathrm{p}_{\mathrm{T}}$ distribution in the 2 jets-2tags region. The latter distribution, fitted in a t't-enriched region, is used to constrain this background. The distributions used in the fit are shown in Figure 8.

Several systematic uncertainties are taken into account; the dominating ones regard the trigger and lepton identification efficiencies.

The measured cross section for $\mathrm{tW}$ production is found to be

$$
\sigma_{\mathrm{tW}}=63.1 \pm 1.8 \text { (stat) } \pm 6.4 \text { (syst) } \pm 2.1 \text { (lumi) pb, }
$$

which is in agreement with the SM predictions.

\section{Rare single top quark production}

In this section we present two new measurements by the CMS Collaboration concerning rare SM processes involving single top quark production: the fist observation of $\mathrm{tZq}$ associated production and the first evidence for $t \gamma q$ associated production.

\subsection{First observation of $\mathrm{tZq}$ associated production}

In order to achieve the first observation of the production of a single top quark in association with a Z boson (tZq associated production), the CMS Collaboration exploited the 2016 and 2017 data sets, corresponding to an integrated luminosity of $77.4 \mathrm{fb}^{-1}$. The analysis [7] target is the leptonic top quark and Z boson decays. Given the tZq topology (see Figure 9), the signal signature requires three charged, isolated leptons, $\mathrm{p}_{\mathrm{T}}$ imbalance, and two jets one of which is $\mathrm{b}$ tagged. The $\mathrm{Z}$ boson candidate is formed taking a pair of opposite sign, same flavor leptons with an invariant mass lying in a mass window of $30 \mathrm{GeV}$ width centered on the world-average $\mathrm{Z}$ boson mass. The top candidate is formed from the $\mathrm{b}$ tagged jet, the $\mathrm{p}_{\mathrm{T}}$ imbalance and the lepton not forming the $\mathrm{Z}$ candidate. 

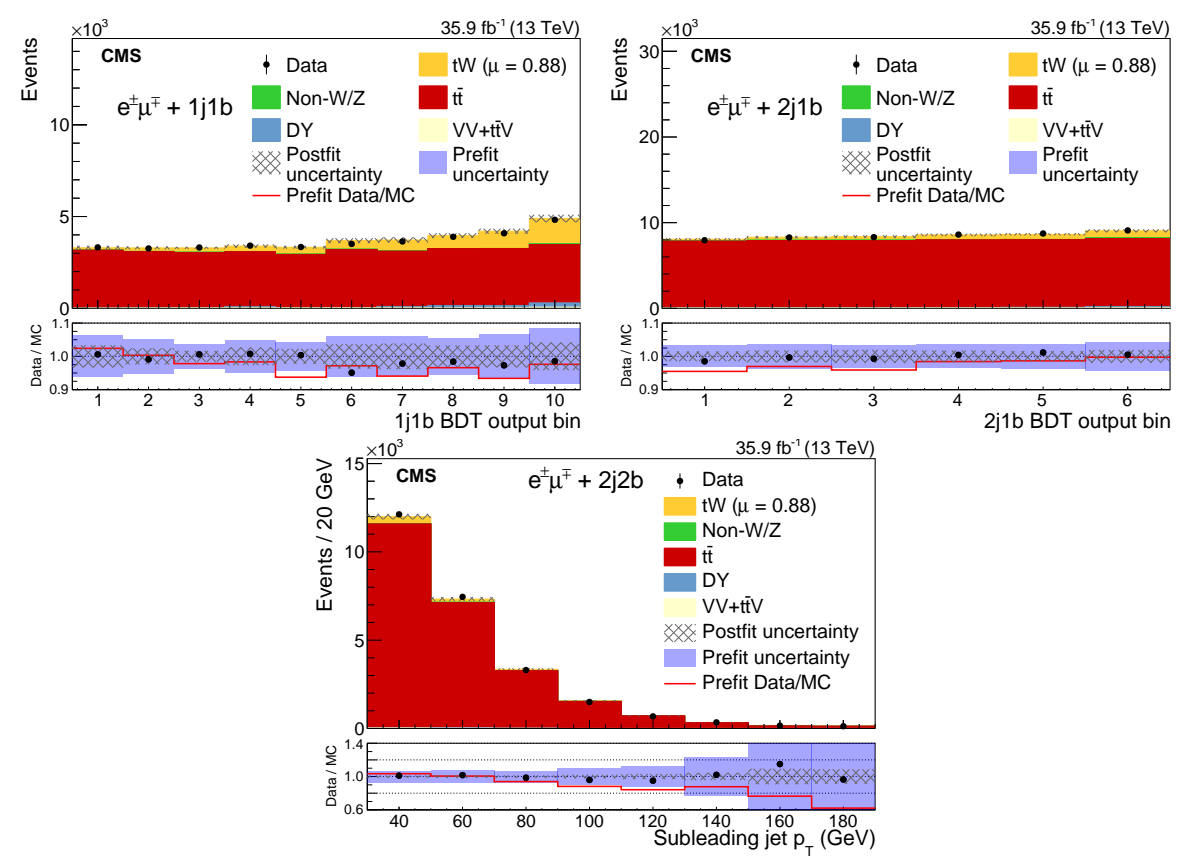

Figure 8: $\mathrm{BDT}_{1 \mathrm{j}-1 \mathrm{~b}}$ output (upper left panel); $\mathrm{BDT}_{2 \mathrm{j}-1 \mathrm{~b}}$ output (upper right panel); subleading jet $\mathrm{p}_{\mathrm{T}}$ in the 2 jets-2tags region (lower panel).

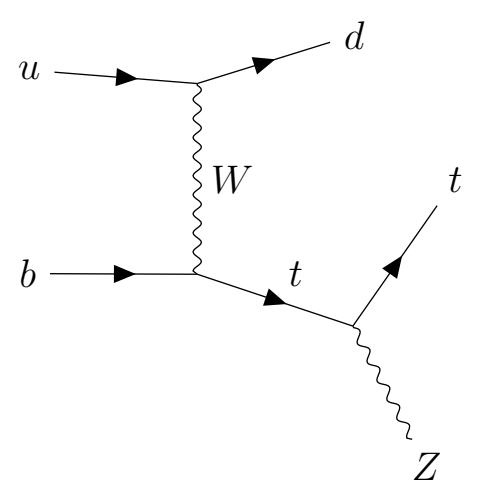

Figure 9: One possible Feynman diagram for the $\mathrm{tZq}$ associated production.
This search crucially depends on efficiently distinguishing leptons originating from the decay of electroweak bosons from the so-called nonprompt leptons (genuine leptons produced in hadron decays, jets misidentified as leptons, etc.). The reach of previous analyses by CMS [8] was severely limited by the relative contribution from nonprompt lepton background and by the uncertainty in its prediction. Taking this into account, BDTs are set up to maximally discriminate between prompt and nonprompt leptons. The BDTs exploit the properties of the jet closest to the lepton in the $\eta-\phi$ space, the lepton relative isolation, the impact parameters with respect to the primary vertex, etc. A tight requirement is placed on the BDT output resulting in a selection efficiency improved by 12 (8) \% for electrons (muons) with respect to the previous CMS analysis. The BDTs are also used to estimate the nonprompt lepton background. Leptons that pass the aforementioned BDT selection criteria are referred to as "tight leptons", while "loose leptons" are a combined set of tight leptons and leptons that pass looser criteria. Data events containing exactly three loose leptons, where at least one of them fails to be a tight lepton, are reweighted with the probability for a loose nonprompt lepton to be a tight nonprompt lepton ("tight-to-loose" ratio method) to obtain a sample used to estimate the nonprompt lepton background.

Events are divided into three signal regions (SRs) based on the number of jets and $\mathrm{b}$ tagged jets. Events with a total of two or three jets, one of which is $b$ tagged, form the SR-2/3j- $1 b$, which 
contains most of the $\mathrm{tZq}$ events. Events with four or more jets, one of which is $\mathrm{b}$ tagged, form the SR-4j-1b, while SR-2b contains events with two or more b tagged jets. In each of these categories, a dedicated BDT is trained to extract the $\mathrm{tZq}$ signal. A simultaneous ML fit to the BDT distributions (shown in Figure 10) is performed to measure the $\mathrm{tZq}$ associated production cross section.

Several systematic uncertainties are taken into account, the uncertainty on the nonprompt lepton background normalization being the dominant one.

The measured product of the cross section and branching fraction for the $\mathrm{tZq}$ associated production is found to be

$$
\sigma_{\mathrm{tZq}} \mathfrak{B}\left(Z \rightarrow \ell^{+} \ell^{-}\right)=111 \pm 13(\text { stat })_{-9}^{+11}(\text { syst }) \mathrm{fb}
$$

in agreement with the SM prediction. The significance of this measurement is well above five standard deviations, resulting in the first observation of the $\mathrm{tZq}$ associated production.

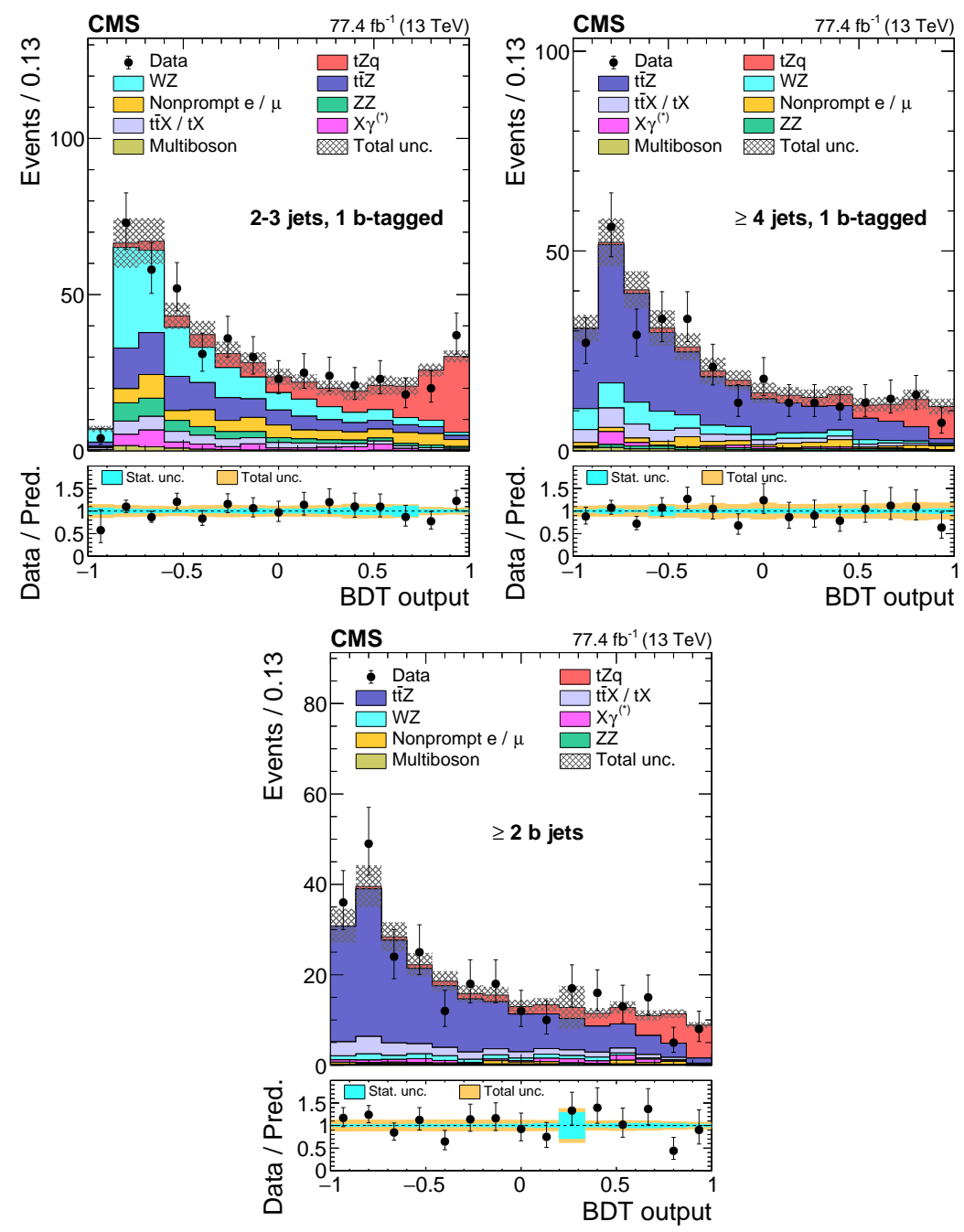

Figure 10: BDT output in the SR-2/3j-1b (upper left panel); SR-4j-1b (upper right panel); SR-2b (lower panel). 


\subsection{First evidence for $t \gamma q$ associated production}

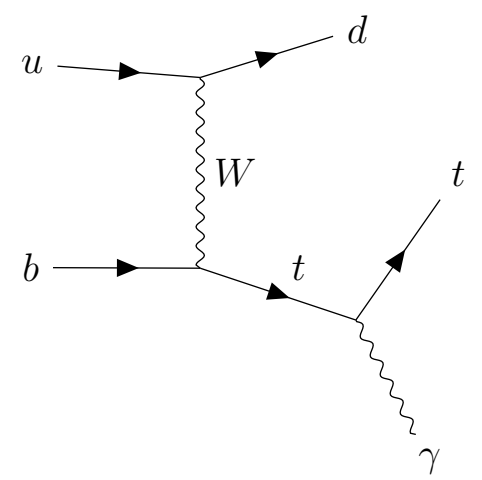

Figure 11: One possible Feynman diagram for the $\mathrm{t} \gamma \mathrm{q}$ associated production.

Finally, the CMS Collaboration produced the first evidence for the production of a single top quark in association with a photon ( $\mathrm{t} \gamma \mathrm{q}$ associated production). The analysis [9] targets the muon decay channel for the top quark since it enables a good signal selection efficiency with low background contamination. The final state includes possible contributions from $\mathrm{W} \rightarrow \tau v_{\tau}$ where the $\tau$ decays to $\mu v_{\mu} v_{\tau}$. Given the $\mathrm{t} \gamma \mathrm{q}$ topology (see Figure 11), the signal signature is one isolated muon, $\mathrm{p}_{\mathrm{T}}$ imbalance, one isolated photon, and two jets one of which is $b$ tagged. Events containing additional muons are rejected.

In order to separate signal from background, a multivariate classification using a BDT is performed. Important variables used to achieve this are the $\eta$ of the light flavor jet, the $\eta$ of che muon candidate, the distance in the space $\eta-\phi$ between the light flavor jet and the photon, etc. The BDT output distribution is parameterized as

$$
F(x)=C_{\mathrm{sig}} S_{\mathrm{sig}}(x)+C_{\mathrm{ti}} S_{\mathrm{tt}}(x)+C_{\mathrm{W} \gamma \mathrm{j}} S_{\mathrm{W} \gamma \mathrm{j}}(x)+C_{\mathrm{Z} \gamma \mathrm{j}} S_{\mathrm{Z} \gamma \mathrm{j}}(x)+C_{\mathrm{misid}} S_{\mathrm{misid}}(x)+C_{\mathrm{B}} S_{\mathrm{B}}(x),
$$

where $x$ is the BDT output, $S_{\mathrm{sig}}(x), S_{\mathrm{tit}}(x), S_{\mathrm{W} \gamma \mathrm{j}}(x), S_{\mathrm{Z} \gamma \mathrm{j}}(x), S_{\text {misid }}(x)$, and $S_{\mathrm{B}}(x)$ are the templates for signal, $\mathrm{t} t+\gamma, \mathrm{W} \gamma+$ jets, $\mathrm{Z} \gamma+$ jets, misidentified photon backgrounds and other minor backgrounds, while the quantities $C_{i}$ are the corresponding normalizations. The different templates $S_{i}(x)$ are taken from simulation except for $t \bar{t}+\gamma$ and misidentifed photons. The former is estimated from data using a control region defined by requiring exactly two $b$ tagged jets and keeping all the other selection criteria the same; the latter is estimate from data measuring the $\mathrm{p}_{\mathrm{T}}$-dependent probability for a jet to be reconstructed as a photon. In order to extract the signal cross section, a simultaneous ML fit is performed on the BDT distributions in the signal region and in the $\mathrm{t} t+\gamma$ control region (see Figure 12).

Several systematic uncertainties are taken into account, the uncertainties on jet energy scale (JES), jet energy resolution (JER) and signal modelling being the dominant ones.

The measured product of the cross section and branching fraction for the $t \gamma q$ associated production is found to be

$$
\sigma_{\mathrm{t} \gamma \mathrm{q}} \mathfrak{B}\left(t \rightarrow \mu v_{\mu} b\right)=115 \pm 17 \text { (stat) } \pm 30 \text { (syst) fb, }
$$

in agreement with the SM prediction. An excess of events above the expected background is observed with a $p$-value of $4.27 \times 10^{-6}$, corresponding to a significance of 4.4 standard deviations, making this measurement the first evidence for the $t \gamma q$ associated production.

\section{Conclusions}

The CMS Collaboration has performed several single top quark measurements, both inclusively and differentially, as well as searches for rare SM processes. All the measurements are in 
agreement with the SM predictions within uncertainties. Most of the analyses are still systematicsdominated, but an improvement in the experimental uncertainties is expected exploiting the full Run2 data set and more.

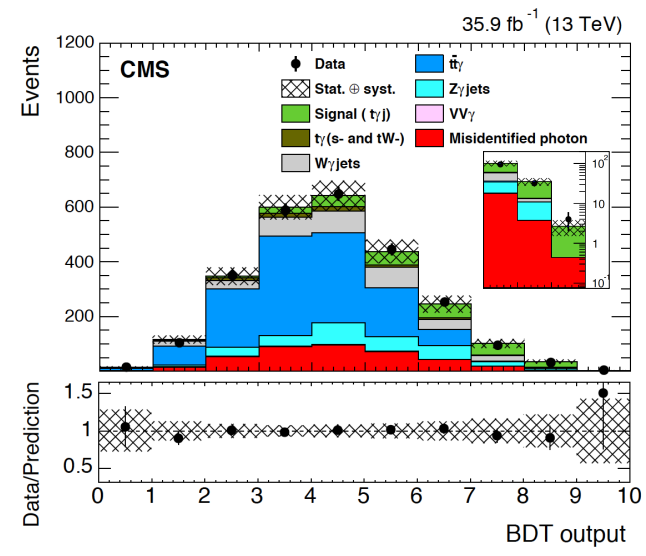

Figure 12: BDT output distribution for data and SM predictions in the signal region.

\section{References}

[1] CMS Collaboration, Measurement of the single top quark and antiquark production cross section in the thannel and their ratio in proton-proton collisions at $\sqrt{s}=13 \mathrm{TeV}$, submitted to PLB, arXiv:1812.10514.

[2] ATLAS and CMS Collaborations, Combination of single-top-quark production cross section measurements and $\left|f_{\mathrm{LV}} \mathrm{V}_{\mathrm{tb}}\right|$ determinations at $\sqrt{s}=7$ and $8 \mathrm{TeV}$ with the ATLAS and CMS experiments, JHEP 05 (2019) 088, arXiv: 1902 . 07158.

[3] ATLAS Collaboration, Fiducial, total and differential cross-section measurements of t-channel single top-quark production in pp collisions at $8 \mathrm{TeV}$ using data collected by the ATLAS detector, Eur. Phys. J. C 77 (2017) 531, arXiv: 1702.02859.

[4] CMS Collaboration, Measurement of differential cross sections and charge ratios for t-channel single top quark production at $13 \mathrm{TeV}$, CMS-PAS-TOP-17-023, 2017.

[5] S. Schmitt, TUnfold: An algorithm for correcting migration effects in high energy physics, JINST 7 (2012) T10003, arXiv:1205.6201.

[6] CMS Collaboration, Measurement of the production cross section for single top quarks in association with $W$ bosons in proton-proton collisions at $\sqrt{s}=13 \mathrm{TeV}$, JHEP 10 (2018) 117, arXiv:1805.07399.

[7] CMS Collaboration, Observation of single top quark production in association with a Z boson in proton-proton collisions at $\sqrt{s}=13 \mathrm{TeV}$, Phys. Rev. Lett. 122 (2019) 132003, arXiv:1812.05900.

[8] CMS Collaboration, Measurement of the associated production of a single top quark and a $Z$ boson in pp collisions at $\sqrt{s}=13 \mathrm{TeV}$, Phys. Lett. B 779 (2018) 358, arXiv: 1712.02825.

[9] CMS Collaboration, Evidence for the associated production of a single top quark and a photon in proton-proton collisions at $\sqrt{s}=13 \mathrm{TeV}$, PRL 121 (2018) 121802, arXiv: 1808.02913. 